\title{
Large-amplitude in-plane atomic vibrations in strained graphene monolayer: bushes of nonlinear normal modes
}

\author{
G. M. Chechin ${ }^{1,2, \dagger}$, D. S. Ryabov'², S. A. Shcherbinin ${ }^{2}$ \\ †gchechin@gmail.com
}

${ }^{1}$ Southern Federal University, Department of physics, Zorge Str., 5, Rostov-on-Don, 344090, Russia

${ }^{2}$ Southern Federal University, Institute of physics, Stachki Ave., 194, Rostov-on-Don, 344090, Russia

Conventional normal modes are independent of each other in the framework of harmonic approximation. When small anharmonic terms in the Hamiltonian are taken into account, these dynamical objects are only approximate and one can speak about their interactions. In our previous works, the theory of bushes of nonlinear normal modes has been developed for dynamical systems with discrete symmetry groups. With the aid of this theory it is possible to find some exact solutions beyond the harmonic approximation. Each bush is an invariant manifold corresponding to a collection of $m$ nonlinear normal modes, where $m$ is the bush dimension. During the evolution of a given bush, this collection conserves, but amplitudes of the modes, entering the bush, change in time. Previously, we proved with the aid of group-theoretical methods, that in graphene under uniform strain (space symmetry group $P 6 \mathrm{~mm}$ ) there can exist 4 bushes with $m=1,14$ bushes with $m=2,1$ bush with $m=3$, 6 bushes with $m=4$, and etc. In this paper, we study some low-dimensional bushes in graphene using ab initio calculations based on the density functional theory. The amplitude-frequency dependencies of one-dimensional bushes are found. The excitation transfer between nonlinear vibrational modes of different symmetry that belong to the same bush is investigated.

Keywords: graphene, group-theoretical methods, nonlinear normal modes, DFT simulations.

\section{Introduction}

The last decades have been marked by intensive studies of nonlinear atomic vibrations in periodic structures, especially, in crystal lattices. One type of such vibrations represents discrete breathers. A recent review on this topic can be found in [1]. There is a large number of papers devoted to studying discrete breathers in graphene [2-6]. In the present work, we investigate another type of nonlinear vibrations in crystal lattice, which, in contrast to discrete breathers, are delocalized dynamical objects.

Traditionally, harmonic approximation is used as a first step in studies of nonlinear dynamics of classical $N$-particle Hamiltonian systems. In this way, one can introduce conventional (linear) normal modes which, being exact solutions in the framework of this approximation, are independent from each other. If small anharmonic terms in the Hamiltonian are taken into account, normal modes turn out to be only approximate solutions. Therefore, the following natural question arises: "Do there exist any exact solutions in nonlinear dynamical systems beyond the harmonic approximation?"

Since any crystal can be characterized by a space group, we are interested in exact vibrational solutions dictated by this symmetry. It occurs $[7,8]$, that such "symmetrydetermined" solutions do exist and we call them bushes of nonlinear normal modes (NNMs). The general theory of bushes of NNMs in systems with discrete symmetries was developed in [7-9]. Bushes of vibrational modes in different physical systems with different point and space symmetries were studied in [7-20].

Every bush is characterized by some subgroup $G_{j} \subset G_{0}$ of the symmetry group $G_{0}$ of dynamical system in its equilibrium state (or a subgroup of its Hamiltonian). We call $G_{0}$ "parent symmetry group". The possibility of a bush to exist as exact dynamical regime is provided by some symmetryrelated selection rules for excitation transfer between modes of different symmetry [7]. In particular, a vibrational mode with higher symmetry cannot excite any mode with lower symmetry: excitation transfers from a given mode to that with higher symmetry group independent of specific types of interparticle interactions in the considered physical system.

Construction of the bushes of NNMs can be done with the aid of group-theoretical methods based on the apparatus of irreducible representations (irreps) of the symmetry groups [21].

Each bush represents a full collection of nonlinear normal modes which are connected by "force" interactions [8] and the number of these modes $(m)$ is the dimension of the given bush. It is essential that bushes with small dimensions (for example, with $m=1,2,3,4, \ldots$ ) can be excited in many physical systems with discrete symmetry. One-dimensional bushes $(m=1)$ describe time-periodical motion while bushes whose dimension $m>1$ describe quasi-periodical motion with $m$ basis frequencies in the Fourier spectrum.

We speak about stability of the given bush, if the collection of its modes is conserved in time. Nevertheless, the bush represents a dynamical object since amplitudes of these 
modes change during the time evolution. Every bush can be considered as an individual Hamiltonian system, whose dimension is less than the whole dimension of the considered system. The energy of the initial excitation turns out to be trapped in the given bush.

If the bush with the symmetry group $G_{j}$ loses its stability, when we increase the energy of initial excitation, it transforms into another bush with larger dimension and with lower symmetry group $\widetilde{G}_{j} \subset G_{j}$.

Some group-theoretical results on small-dimensional vibrational bushes in graphene were published in our previous paper [20], which was devoted to geometrical aspects of bushes of NNMs. We discussed their structure, i.e. the collection of nonlinear vibrational modes entering bushes and displacement patterns associated with these modes. In the present paper, we discuss dynamical aspects of vibrational bushes in graphene. Namely, we consider the evolution of the modes of the given bush in time, in particular, the transfer of excitation from the initially excited mode ("root mode") to the other modes ("secondary modes") of the bush.

Our discussion of the bush dynamics is based on the computational methods of the density functional theory [22] realized in software package Quantum Espresso [23]. Quantum-mechanical equations by Kohn and Sham are used in the Quantum Espresso code for describing dynamics of electrons, while classical equations are solved for nuclei with forces generated by electron shells at any time step. For solving Kohn-Sham equations, the basis of plane waves was used with maximal energy determined by cutoff energy $E_{\text {cutoff }}=50$ Hartree and time step was $0.2419 \mathrm{fs}$.

The paper is organized as follows. Section 2 is devoted to some group-theoretical results which were not presented in the previous publication [20]. In Sec. 3, we discuss onedimensional bushes, while in Sec. 4, we consider twodimensional bushes in graphene monolayer under uniform strain (space group $P 6 \mathrm{~mm}$ ). In Conclusion, we summarize the main results.

\section{Bushes of vibrational nonlinear normal modes}

A brief review of the group-theoretical methods for construction of bushes of nonlinear normal modes was presented in the previous paper [20], while full description of these methods can be found in $[7,21]$.

As was already discussed, an $m$-dimensional bush $B_{j}(t)$ consists of $m$ individual NNMs interacting with each other. It can be written as follows:

$$
B_{j}(t)=\sum_{k=1}^{m} c_{j k}(t) \boldsymbol{\varphi}_{k}
$$

Here $\varphi_{k}$ are $N$-dimensional vectors, where $N$ is the number of degrees-of-freedom in an extended cell (EC) of the considered crystal. In general, this cell is larger than the primitive cell by an integer factor $s$. The vectors $\varphi_{k}$ represent basis vectors of different irreducible representations of the parent space group $G_{0}$ (for the graphene monolayer $G_{0}=P 6 \mathrm{~mm}$ ). Each $\boldsymbol{\varphi}_{k}$ determines a certain displacement pattern with its own symmetry group $G_{k} \subseteq G_{0}$. Some examples of such patterns can be seen in Fig. 1. Every pattern represents a snapshot of the vibrational picture of the graphene monolayer. All arrows in each of Fig. 1 possess equal length, but different directions. They determine the displacements of atoms from their equilibrium positions. Vibrational time-evolution of these displacements is describes by the coefficients $c_{j k}(t)$ in Eq. (1), which are different for different modes (index $k$ ) and different bushes (index $j$ ).

Speaking about the stability of the given bush we mean that the set of its basis vectors $\varphi_{k}, k=1 . . m$ conserves in time. On the other hand, the time-dependent coefficients $c_{j k}(t)$ change and their behavior describes evolution of the given bush.

Discussing the vector collection $\left\{\boldsymbol{\varphi}_{k}, k=1 . . m\right\}$ of the $m$-dimensional bush, we deal with its geometrical aspect. In mathematical sense each bush represents a certain invariant manifold ${ }^{1}$ decomposed in the basis vectors of the irreps of the parent symmetry group. These invariant manifolds we find with the help of some specific group-theoretical methods [21]. On the other hand, considering the time-behavior of the coefficients $c_{j k}(t)$ we deal with dynamical aspects of the bush.

The displacement pattern corresponding to a given bush $B_{j}(t)$ at any fixed time $t$ represents a certain superposition of $m$ delocalized nonlinear vibrational modes $c_{j k}(t) \boldsymbol{\varphi}_{k}$. For the case of essentially weak nonlinearity

$$
c_{j k}(t)=c_{j k}^{0} \cos \left(\omega_{j k} t+\phi_{j k}^{0}\right),
$$

and, therefore, each nonlinear normal mode transforms to a certain conventional linear normal mode.

As was already mentioned in Introduction, the existence of bushes as exact small-dimensional dynamical objects can be explained by existence of certain selection rules for excitation transfer between vibrational modes of different symmetry. Indeed, the modes entering a given bush, in general, possess different symmetry groups. The symmetry of the whole bush is the intersection of all these groups of individual modes.

Let us excite a certain vibrational mode with symmetry group $G_{j}$. In accordance with the above-mentioned selection rules, the excitation from this "root" mode can transfer only to those modes whose symmetry is greater or equal to $G_{j}{ }^{2}$ Namely, these modes are called "secondary" modes. As a result, the symmetry group of the whole bush will be also equal to $G_{j}$. Thus, if we excite an arbitrary vibrational mode, a certain bush will appear. However, the dimension of this bush can be sufficiently large if we initially excite the mode with sufficiently low symmetry group $G_{j}$. On the contrary, if the root mode possesses high symmetry ${ }^{3}$ the bush can be of small dimension.

In [20] we found that in graphene monolayer, whose symmetry group in equilibrium state is $G_{0}=P 6 \mathrm{~mm}$, only 4 one-dimensional, 14 two-dimensional, 1 three-dimensional and 6 four-dimensional vibrational bushes, corresponding to the points of high symmetry in the Brillouin zone, can be excited.

1 This manifold is invariant with respect to the time evolution of the system.

2 These rules are not confined to this single statement!

3 This means that group $G_{j}$ contains large number of symmetry elements. 

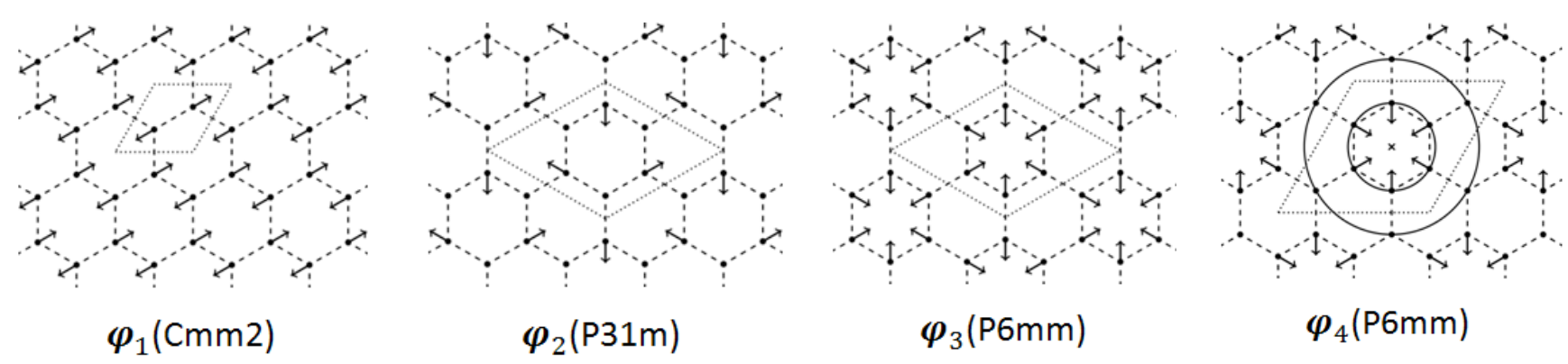

Fig. 1. Nonlinear normal modes $\varphi_{1}, \varphi_{2}, \varphi_{3}, \varphi_{4}$ which are one-dimensional bushes.

Note, that a given bush $B_{j}(t)$, which appears as a result of excitation of the root mode only, can be also generated by excitation of all its modes simultaneously, in accordance with Eq. (1), by assigning the initial values $c_{j k}\left(t_{0}\right), \dot{c}_{j k}\left(t_{0}\right)$ in Eq. (1) for all modes $k=1 . . m$. However, generation of a given bush by exciting only one mode seems to be the most natural way of the bush excitation.

Let us emphasize once more that the bush structure as a certain collection of modes is determined by its symmetry group only and does not depend on the specific interparticle interactions in the physical system. As a consequence, we can reveal vibrational bushes of identical structures in physical systems with different type of interparticle interactions, but with the same symmetry group $G_{0}$ in equilibrium state. On the other hand, dynamics of the bush, i.e. explicit form of the time-dependent functions $c_{j k}(t)$, essentially depends on these interactions.

\section{One-dimensional bushes in graphene monolayer}

We have found that only 4 one-dimensional bushes can exist in graphene. Their displacement patterns are presented in Fig. 1. Extended cells (EC) of vibrational state are shown in this figure by dotted lines.

Let us consider the bush $B_{3}(t)$ with the symmetry group $G_{3}=P 6 m m$. Only one term, $c_{31}(t) \varphi_{31}$, corresponds to it in Eq. (1). Arrows show the values and directions of atomic displacements at a certain fixed instant. The time-evolution of these displacements are defined by only one function $c_{31}(t)$. Here, $\varphi_{31}$ is a 12 -dimensional vector since EC for this bush contains 6 atoms and each of them possesses 2 degrees of freedom, describing its in-plane vibrations. The bush $B_{3}(t)$ describes synchronic vibrations of all atoms of the graphene monolayer. If the function $c_{31}(t)$ is equal to zero, at a certain moment, all atoms pass through their equilibrium positions, and further evolution leads to a change of directions of atomic displacements to opposite ones.

Atomic vibrations described by one-dimensional bushes are time-periodic. Moreover, every one-dimensional bush describes one-parametric dynamical regime since positions of all graphene atoms are determined, at any time $t$, by only one parameter $c_{31}(t)$.

To avoid misunderstandings, let us note that we sometimes use the term "mode" not only for the full expression $c(t) \varphi$, but also for the vector $\varphi$, which determines the displacement pattern of a given mode.
Let us note, that all arrows for the bush $B_{3}(t)$ in Fig. 1 (as well as in all similar figures below), being different in directions are equal in length.

Next, we consider the properties of nonlinear vibrations described by the time-dependent function $c_{31}(t)$. If a certain one-dimensional bush appears as a result of a certain choice of the initial conditions for computer modeling, time-evolution of all atoms must be identical, while in the opposite case timeevolution of different atoms will be different.

In Fig. 2, we present nonlinear vibrations of one carbon atom of graphene for the bush $B_{3}(t)$ since the same timeevolution corresponds to all other atoms. It can be seen from this figure, that these oscillations are nonlinear.

In Fig. 3, amplitude-frequency dependency of atomic nonlinear oscillations is shown for the graphene monolayer under uniform strain of $5 \%$ and $10 \%$. Fig. 3a, corresponding to the bush $B_{3}(t)$, whose displacement pattern is shown in Fig. 1, demonstrates hard nonlinearity of atomic vibrations since the frequency increases with increasing amplitude.

In Fig. 3b, for the graphene monolayer under the same uniform strains, we present the amplitude-frequency dependency of atomic oscillations, corresponding to the bush $B_{1}(t)$, whose displacement pattern is shown in Fig. 1 . In contrast to the above discussed bush $B_{3}(t)$, the bush $B_{1}(t)$ demonstrates soft type of nonlinearity (the frequency decrease with amplitude increasing). The amplitudefrequency dependencies for two other one-dimensional bushes, $B_{2}(t)$ [soft nonlinearity] and $B_{4}(t)$ [hard nonlinearity] are expressed very weakly.

It is interesting to note that in the bushes $B_{1}(t)$ and $B_{3}(t)$ all graphene atoms vibrate, while in the bushes $B_{2}(t)$ and $B_{4}(t)$ some atoms are motionless (see Fig. 1).

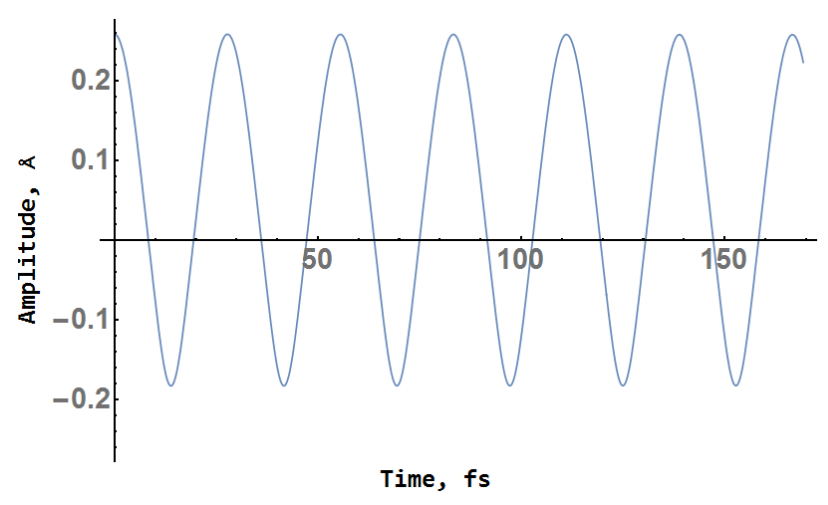

Fig. 2. Nonlinear atomic oscillations for the bush $B_{3}(t)$. 


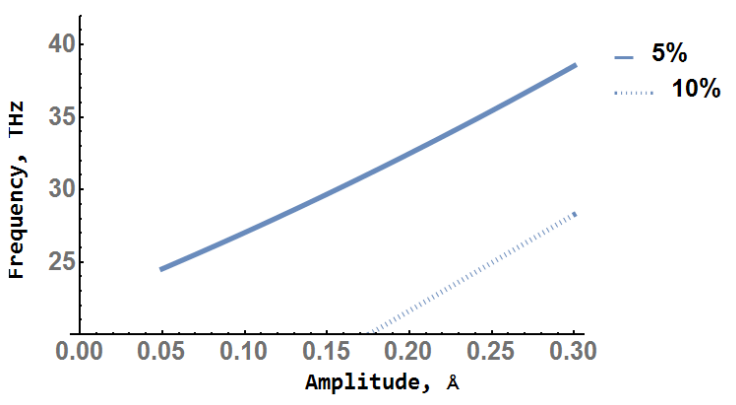

a

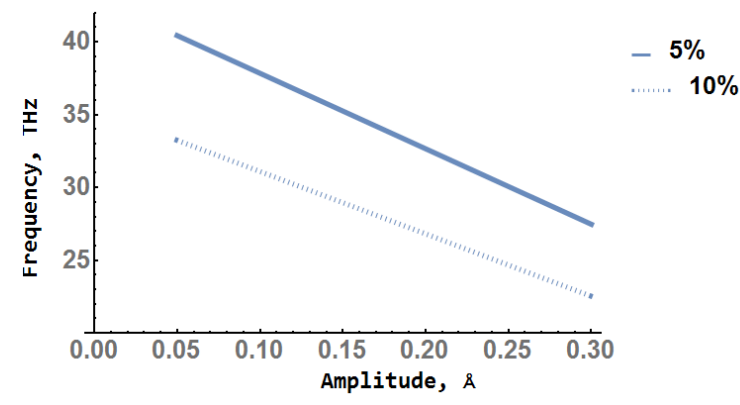

b

Fig. 3. Amplitude-frequency dependencies of atomic nonlinear oscillations for the one-dimensional bushes: a) $B_{3}(t)$; b) $B_{1}(t)$.

\section{Two-dimensional bushes}

Let us consider some two-dimensional bushes in graphene with different multiplications $s$ of the cell in the vibrational state (EC).

Among 14 two-dimensional bushes there are only 3 with multiplication number $s=4$ : the bush $B_{5}(t)$ with space group $P 6, B_{6}(t)$ with group $P 3 m 1$ and $B_{7}(t)$ with group $P 31 m$.

1) We begin with the bush

$$
B_{5}(t)=\mu(t) \varphi_{5}+v(t) \varphi_{4} .
$$

Here, we replace the functions $c_{55}(t)$ and $c_{54}(t)$ in Eq. (1) by the functions $\mu(t)$ and $v(t)$, respectively, to avoid unnecessary indexes.

Displacement pattern for the root mode $\varphi_{5}$ of this bush can be seen in Fig. 4, where position of one six-fold rotation axis of the infinite set of such axes is marked with a cross. The motion of six atoms of the first "coordinate sphere" (in twodimensional case it is a circle) is characterized by the so-called "tilting mode". This means that they rotate by a certain angle around the above axis, stop for a moment and then start to rotate in the opposite direction. On the other hand, six atoms of the second coordination sphere remain immobile.

However, this mode, being excited, cannot exist as an individual nonlinear vibrational mode. Indeed, another (secondary) mode, $\varphi_{4}$, turns out to be involved in the oscillatory process as a result of the excitation of the root mode $\varphi_{5}$ at the initial moment of time.

The displacement pattern for the above mode $\varphi_{4}$ is given in Fig. 1. If this mode is initially excited, then it would exist indefinitely in time as an independent dynamic object which is one-dimensional bush $B_{4}(t)$, considered in the previous section.
It can be seen from Fig. 1 that, similar to the case of the mode $\varphi_{5}$, all atoms of the second coordination sphere are immobile, while motion of atoms of the first coordination sphere is described by a "breathing" mode: they simultaneously move towards the position of the cross and then backwards from it.

Thus, the mode $\varphi_{4}$ can exist independently of the mode $\varphi_{5}$ (as well as of all other modes!), i.e. it represents by itself onedimensional bush $B_{4}(t)$. On the other hand, the mode $\varphi_{5}$ can not exist without the secondary mode $\varphi_{4}$ that is involved into the vibrational process because of interaction with the root mode $\varphi_{5}$. This is the main property of every bush of nonlinear normal modes. Note, the symmetry group of the secondary mode $\varphi_{4}$ is $P 6 \mathrm{~mm}$, while that of the root mode $\varphi_{5}$ is $P 6$, which is a subgroup of the group $P 6 \mathrm{~mm}$.

Speaking about the two-dimensional bush $B_{5}(t)$ from Eq. (3), we should keep in mind that it is an exact solution of the nonlinear physical system with symmetry group $P 6 \mathrm{~mm}$ for arbitrary functions $\mu(t)$ and $v(t)$ : this linear combination survives in time without involvement of any other vibrational modes in graphene.

A similar situation occurs in studying interactions between rotational and vibrational nonlinear modes in molecules. Indeed, in the framework of harmonic approximation conventional normal modes are independent from each other. However, they start to interact when we take into account some anharmonic terms of Hamiltonian. The breathing mode can exist without excitation of rotating mode. However, excitation of rotating mode leads to excitation of the breathing mode. In the last case, twodimensional bush appears in dynamics of polyatomic molecule, and the rotating mode is the root mode of this bush.
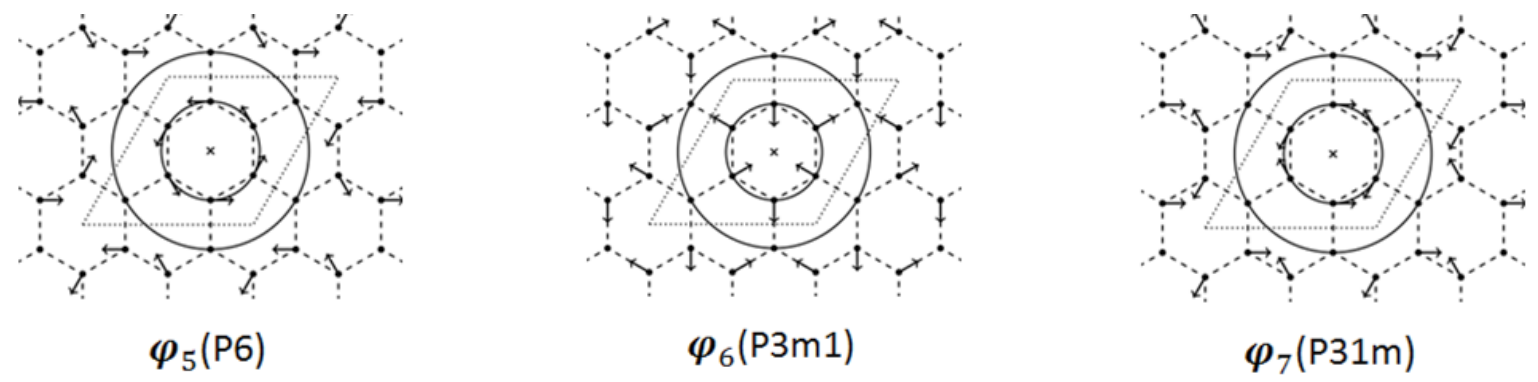

Fig. 4. Displacement patterns for the root modes $\varphi_{5}, \varphi_{6}, \varphi_{7}$ of the bushes $B_{5}(t), B_{6}(t), B_{7}(t)$ respectively. 
Using computational methods of the density functional theory [23], we have verified the above-discussed results of the group-theoretical analysis. At the initial instant, only root mode $\mu(t) \varphi_{5}$ is excited with a certain value of $\mu(0) \varphi_{5} \neq 0$, while the value of the secondary mode $v(t) \boldsymbol{\varphi}_{4}$ is equal to zero $v(0)=0$. One can see that during the time-evolution $(t>0)$ the secondary mode $\varphi_{4}$ appears, but it amplitude is essentially small compared to that of the root mode. For example, in Fig. 5a, for the case $\mu(0)=0.381 \AA$, the amplitude of the secondary mode $v(t)$ is a 20 times less than that of the root mode.

2) Next, let us consider the bush $B_{6}(t)$ with space group $P 3 \mathrm{~m} 1$. The displacement pattern of its root mode $\varphi_{6}$ is presented in Fig. 4. The secondary mode of this bush is $\varphi_{4}$ whose displacement pattern is given in Fig. 1. Thus, the bushes $B_{5}(t)$ and $B_{6}(t)$ possess one and the same secondary mode, but these bushes differ from each other by their root modes. For both bushes $B_{5}(t)$ and $B_{6}(t)$ atoms on the second coordination sphere are immobile, while atoms belonging to the first coordination sphere move in different ways. Indeed, unlike the titling motion in the mode $\varphi_{5}$, the mode $\varphi_{6}$ describes some type of vibration which is shown by corresponding arrows in Fig. 4.

Time-evolution of the root and secondary modes of the bush $B_{6}(t)$ in graphene under uniform $5 \%$ strain for two different initial values of the root mode is presented in Fig. 5b.

3) Displacement pattern of the root mode $\varphi_{7}$ of the bush $B_{7}(t)$ is presented in Fig. 4. Similar to the case of the bushes $B_{5}(t)$ and $B_{6}(t)$, the secondary mode of the bush $B_{7}(t)$ is $\varphi_{4}$. The atoms of the second coordination sphere are also immobile in vibrational process describing by the bush $B_{7}(t)$, while the atoms of the first coordination sphere move in a way, different from that of the bushes $B_{5}(t)$ and $B_{6}(t)$ depicted in Fig. 4.

Time-evolution of the bush $B_{7}(t)$ in graphene under uniform 5\% strain is presented in Fig. 5c. Note, that the secondary mode, being small at the beginning of motion, becomes comparable in order of magnitude with the root mode as time goes.

\section{Conclusion}

In this paper, large-amplitude in-plane vibrations of carbon atoms of graphene monolayer under uniform strain (space group $P 6 \mathrm{~mm}$ ) are discussed. These oscillations are described in the framework of the theory of bushes of nonlinear normal modes, which represent exact solutions for any nonlinear physical system with discrete symmetry. We discuss both the structure of low-dimensional bushes, which can be obtained with the aid of group-theoretical methods, as well as dynamics of these bushes by means of ab initio calculations based on the density functional theory. We have obtained full confirmation of the group-theoretical results by such $a b$ initio computer modeling.

One application of our results can be found in papers [24, 25], where discrete breathers are constructed by imposition of certain bell-shaped functions on the displacement patterns of one-dimensional bushes in graphene.

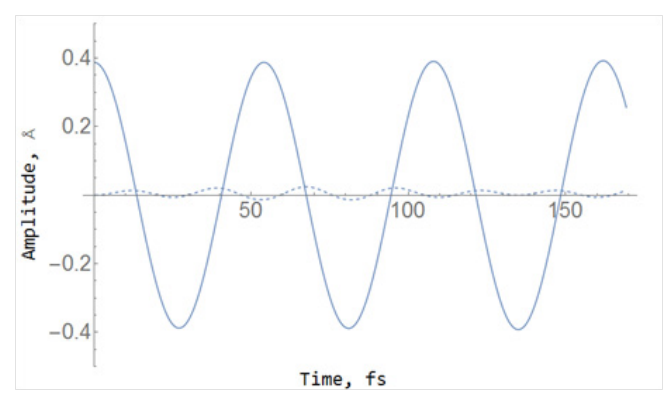

a

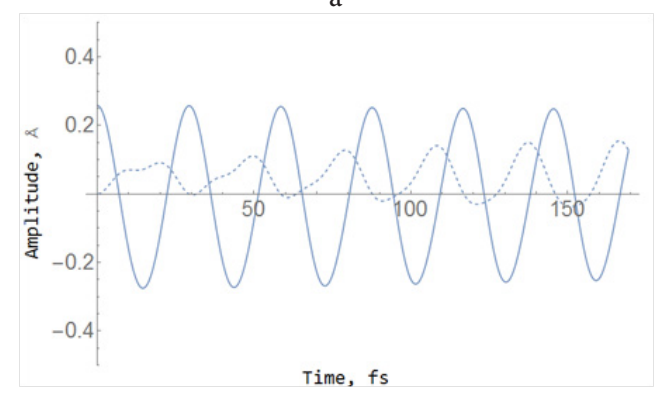

b

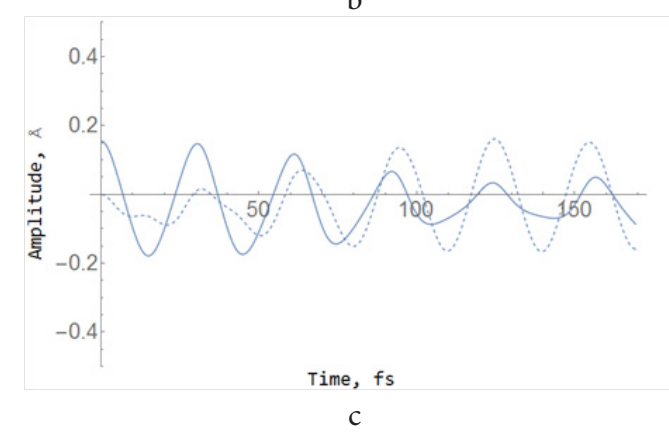

Fig. 5. Time-evolution of the root (solid line) and secondary (dashed line) modes of the two-dimensional bushes for $5 \%$ uniform strain of the graphene monolayer:

$\begin{array}{lll}\text { a) } B_{5}(t)=\mu(t) \boldsymbol{\varphi}_{5}+v(t) \boldsymbol{\varphi}_{4} & {[\mu(0)=0.381 \AA,} & v(0)=0] \text {; } \\ \text { b) } B_{6}(t)=\mu(t) \boldsymbol{\varphi}_{6}+v(t) \boldsymbol{\varphi}_{4} & {[\mu(0)=0.254 \AA,} & v(0)=0] ; \\ \text { c) } B_{7}(t)=\mu(t) \boldsymbol{\varphi}_{7}+v(t) \boldsymbol{\varphi}_{4} & {[\mu(0)=0.152 \AA,} & v(0)=0]\end{array}$

Acknowledgments. The authors are sincerely grateful to Profs. V.P. Sakhnenko and N.V. Ter-Oganessian for useful discussions. The authors acknowledge support by the Ministry of Education and Science of the Russian Federation (state assignment grant No. 3.5710.2017/8.9). S. A. Shcherbinin acknowledges financial support by the Russians Science Foundation (Grant No. 14-13-00982).

\section{References}

1. S.V. Dmitriev, E.A. Korznikova, J.A. Baimova, M. G. Velarde. Phys. Usp. 59, 446 (2016).

2. Y. Doi, A. Nakatani. Journal of Solid Mechanics and Materials Engineering 6 (1), 71 (2012).

3. E. Barani, I. P. Lobzenko, E. A. Korznikova, E. G. Soboleva, S. V. Dmitriev, K. Zhou, A.M. Marjaneh. Eur. Phys. J. B 90, 38 (2017).

4. I.P. Lobzenko, I. Evazzade, M.R. Roknabadi, R. I. Makhmutova, S. V. Dmitriev. Letters on materials, 6 (2), 152 - 155 (2016). DOI: 10.22226/2410-3535-2016-2152-155 
5. I. Evazzade, I. P. Lobzenko, E. A. Korznikova, I. A. Ovid`ko, M. R. Roknabadi, S. V. Dmitriev. Phys. Rev. B 95, 035423 (2017).

6. E.A. Korznikova, D. V. Bachurin, S.Y. Fomin, A.P. Chetverikov, S. V. Dmitriev. Eur. Phys. J. B. 90, 23 (2017).

7. V.P. Sakhnenko, G. M. Chechin. Dokl. Akad. Nauk 330, 308 (1993); V.P. Sakhnenko, G. M. Chechin. Phys. Dokl. 38, 219 (1993).

8. G. M. Chechin, V.P. Sakhnenko. Physica D 117, 43 (1998).

9. V.P. Sakhnenko, G. M. Chechin. Dokl. Akad. Nauk 338, 42 (1994); V.P. Sakhnenko, G. M. Chechin. Phys. Dokl. 39, 625 (1994).

10. G. Chechin, D. Sizintsev, O. Usoltsev. Letters on materials 6 (2), 146 - 151 (2016). DOI: 10.22226/2410-3535-20162-146-151

11. G. Chechin, D. Sizintsev, O. Usoltsev. Letters on materials 6 (4), 309 - 316 (2016). DOI: 10.22226/2410-3535-20164-309-316

12. G. Chechin, D. Sizintsev, O. Usoltsev. Comp. Materials Science 138C, 353 (2017).

13. G. M. Chechin, D.S. Ryabov, K.G. Zhukov. Physica D 203, 121 (2005).
14. G. M. Chechin, A.V. Gnezdilov, M. Yu. Zekhtser. Int. J. Non-Linear Mech. 38, 1451 (2003).

15. G. M. Chechin, K.G. Zhukov. Phys Rev E 73, 36216 (2006).

16. G. M. Chechin, D. S. Ryabov. Phys. Rev. E 69, 036202-1 (2004).

17. G.M. Chechin, S.A. Shcherbinin. Commun. in Nonlinear Sci. and Numer. Simulat. 22, 244 (2015).

18. G.M. Chechin, D.S. Ryabov, S.A. Shcherbinin. Phys. Rev. E 92, 012907 (2015).

19. G.S. Bezuglova, G.M. Chechin, P.P. Goncharov. Phys. Rev. E. 84, 036606 (2011).

20. G. M. Chechin, D. S. Ryabov, S. A. Shcherbinin. Letters on materials 6 (1), 9 - 15 (2016). DOI: 10.22226/2410-35352016-1-9-15

21. G. M. Chechin. Comput. Math. Appl. 17 (1-3), 255 (1989).

22. W. Kohn. Rev. Mod. Phys. 71, 1253 (1999).

23. P. Giannozzi et al. J. Phys.: Condens. Matter, 21, 395502 (2009).

24. S. V. Dmitriev, E. A. Korznikova, D. I. Bokij, K. Zhou. Physica Status Solidi B 253 (7), 1310 (2016).

25. E. A. Korznikova, S. Yu. Fomin, S. V. Dmitriev. JETP Letters, 4, 303 (2016). 